PROCEEDINGS OF THE

AMERICAN MATHEMATICAL SOCIETY

Volume 127, Number 9, Pages 2583-2586

S 0002-9939(99)04982-5

Article electronically published on April 9, 1999

\title{
ON GROUPS WITH COMMUTATORS OF BOUNDED ORDER
}

\author{
PAVEL SHUMYATSKY
}

(Communicated by Ronald M. Solomon)

\begin{abstract}
Let $p$ be a prime, $k$ a non-negative integer. We prove that if $G$ is a residually finite group such that $[x, y]^{p^{k}}=1$ for all $x, y \in G$, then $G^{\prime}$ is locally finite.
\end{abstract}

\section{INTRODUCTION}

The aim of this paper is to prove the following

Theorem. Let $q=p^{k}$ be a prime power, $G$ a residually finite group such that $[x, y]^{q}=1$ for all $x, y \in G$. Then $G^{\prime}$ is locally finite.

Of course, the above theorem fails to be true if the assumption of residual finiteness of $G$ is dropped. As is known from the famous negative solutions to the Burnside Problem [1],[6] even groups satisfying the identity $x^{q}=1$ need not be locally finite when $q$ is big enough. In fact, it follows from the Ol'shanskii's theory that for sufficiently big values of $q$ a group $G$ can satisfy the identity $[x, y]^{q}=1$ and have the derived group $G^{\prime}$ non-periodic! (This was communicated to us by V. Obraztsov.)

In view of our result some conjectures naturally arise, in particular it seems very likely that for any non-negative integer $n$ the identity $[x, y]^{n}=1$ on elements of a residually finite group $G$ implies that $G^{\prime}$ is locally finite. Another interesting question is whether under the hypothesis of the above theorem $G^{\prime}$ is necessarily of finite exponent. Note that it follows from our proof that this is so if $G$ is finitely generated.

As the reader can see the paper is quite short. The brevity is achieved by using "heavy weapons"; in particular a deep theorem of Zelmanov on PI Lie algebras (see Theorem 2.1 below) and his solution to the Restricted Burnside Problem.

We say for short that a number is $X$-bounded if it is bounded from above by some function that depends only on parameters from the set $X$. Throughout the paper $p$ denotes a fixed prime and $q=p^{k}$ a fixed power of $p$.

The author wishes to express his thanks to V. Obraztsov for stimulating discussion, to A. Mann for permission to include his short proof of Lemma 3.2, and to the anonymous referee for a number of useful comments on the paper.

Received by the editors November 15, 1997.

1991 Mathematics Subject Classification. Primary 20E26, 20F40; Secondary 20F50.

Key words and phrases. Residually finite group, associated Lie algebra.

This work was supported by FAPDF and CNPq-Brazil. 


\section{On $p$-GRoups AND Their associated Lie AlgEbras}

This section does not contain new results. For the reader's convenience we collect here some necessary definitions and facts on Lie algebras associated with finite $p$ groups. The most adequate for our purposes general reference for the subject is [11].

Let $L$ be a Lie algebra over a field $\mathfrak{k}$. We use the left normed notation: thus if $l_{1}, l_{2}, \ldots, l_{n}$ are elements of $L$, then

$$
\left[l_{1}, l_{2}, \ldots, l_{n}\right]=\left[\ldots\left[\left[l_{1}, l_{2}\right], l_{3}\right], \ldots, l_{n}\right] .
$$

An element $a \in L$ is called ad-nilpotent if there exists a positive integer $n$ such that $[x, \underbrace{a, \ldots, a}_{n \text { times }}]=0$ for all $x \in L$. If $n$ is the least integer with the above property, then we say that $a$ is ad-nilpotent of index $n$. Let $X \subseteq L$ be any subset of $L$. By a commutator in elements of $X$ we mean any element of $L$ that could be obtained from elements of $X$ by means of repeated operation of commutation with an arbitrary system of brackets including the elements of $X$. Denote by $F$ the free Lie algebra over $\mathfrak{k}$ on countably many free generators $x_{1}, x_{2}, \ldots$ Let $f=f\left(x_{1}, x_{2}, \ldots, x_{n}\right)$ be a non-zero element of $F$. The algebra $L$ is said to satisfy the identity $f \equiv 0$ if $f\left(a_{1}, a_{2}, \ldots, a_{n}\right)=0$ for any $a_{1}, a_{2}, \ldots, a_{n} \in L$. In this case we say that $L$ is PI. We are now in a position to quote a theorem of Zelmanov which has numerous important applications to group theory.

Theorem 2.1 (Zelmanov, [11], III(0.4)). Let $L$ be a Lie algebra generated by $a_{1}$, $a_{2}, \ldots, a_{m}$. Assume that $L$ is $P I$ and that each commutator in the generators $a_{1}, a_{2}, \ldots, a_{m}$ is ad-nilpotent. Then $L$ is nilpotent.

In this paper we use the following corollary of the above theorem.

Theorem 2.2. Let $L$ be a Lie algebra over a field $\mathfrak{k}$ generated by $a_{1}, a_{2}, \ldots, a_{m}$. Assume that $L$ satisfies an identity $f \equiv 0$ and that each commutator in the generators $a_{1}, a_{2}, \ldots, a_{m}$ is ad-nilpotent of index at most $n$. Then $L$ is nilpotent of $\{f, n, m, \mathfrak{k}\}$-bounded class.

Proof. Consider the free $m$-generated Lie $\mathfrak{k}$-algebra $F_{m}$ on free generators $f_{1}, \ldots, f_{m}$, and let $T$ be the ideal of $F_{m}$ generated by all values of $f$ on the elements of $F_{m}$ and by all elements of the form $[g, \underbrace{c, \ldots, c}_{n \text { times }}]$, where $g \in F_{m}$ and $c$ is an arbitrary commutator in the $f_{i}$. Then $F / T$ satisfies the hypothesis of Theorem 2.1 and hence is nilpotent of some class $u=u(m, n, f, \mathfrak{k})$. Clearly, $L$ is an image of $F / T$ under the homomorphism induced by the mapping $f_{i} \rightarrow a_{i}$. Hence $L$ is nilpotent of class at most $u$.

Let $G$ be a group and $M$ a subset of $G$. We call an element $x \in G$ a commutator of weight 1 in elements of $M$ if and only if $x \in M$. Now assume that $w \geq 2$ and commutators in elements of $M$ of any weight less than $w$ are already defined. An element $x \in G$ is called a commutator of weight $w$ in elements of $M$ if and only if there exist commutators $x_{1}, x_{2}$ of weights $w_{1}$ and $w_{2}$ in elements of $M$ such that $w_{1}+w_{2}=w$ and $x=\left[x_{1}, x_{2}\right]$. Of course, there can be various ways to represent an element of $G$ as a commutator in elements of $M$. If the set $M$ is not indicated, we use the term "commutator" to mean a commutator of weight 2 in elements of $G$. 
Let $G$ be a finite $p$-group. We denote by $D_{i}=D_{i}(G)$ the $i$-th dimension subgroup of $G$ in characteristic $p$ (see [5], Chapter 8). These subgroups form a central series of $G$ known as the Zassenhaus-Jennings-Lazard series. Set $L(G)=\bigoplus D_{i} / D_{i+1}$. Then $L(G)$ can naturally be viewed as a Lie algebra over the field $\mathbb{F}_{p}$ with $p$ elements. Let $x \in G$, and let $i=i(x)$ be the largest integer such that $x \in D_{i}$. We denote by $\tilde{x}$ the element $x D_{i+1} \in L(G)$.

Lemma 2.3 (Lazard, [7], p. 131). For any $x \in G$ we have $(\operatorname{ad} \tilde{x})^{p}=\operatorname{ad}\left(\tilde{x^{p}}\right)$. In particular, if $x^{q}=1$ then $\tilde{x}$ is ad-nilpotent of index at most $q$.

Let us denote by $L_{p}(G)$ the subalgebra of $L$ generated by $D_{1} / D_{2}$. Fix a positive number $c$, and assume that $G$ is generated by $g_{1}, g_{2}, \ldots, g_{m}$. Let $\rho_{1}, \rho_{2}, \ldots, \rho_{r}$ be the list of all commutators in $g_{1}, g_{2}, \ldots, g_{m}$ of weight at most $c$. Here $r$ obviously is $\{c, m\}$-bounded. The following lemma is straightforward from arguments of Zelmanov given in [11], p. 71.

Lemma 2.4. Let $G$ be a finite p-group. If $L_{p}(G)$ is nilpotent of class $c$, then $G=\left\langle\rho_{1}\right\rangle\left\langle\rho_{2}\right\rangle \ldots\left\langle\rho_{r}\right\rangle$ is the product of cyclic subgroups generated by the $\rho_{i}$ 's.

Again let $F$ be the free Lie algebra over $\mathbb{F}_{p}$ on countably many free generators $x_{1}, x_{2}, \ldots$ Let $f \in F$ be the linearization of the Lie polynomial

$$
[x_{3}, \underbrace{\left[x_{1}, x_{2}\right],\left[x_{1}, x_{2}\right], \ldots,\left[x_{1}, x_{2}\right]}_{q \text { times }}] .
$$

We do not require the explicit form of $f$ but note that $f \neq 0$ (see [2]).

The next lemma follows from the proof of Theorem 1 in the paper of Wilson and Zelmanov [8].

Lemma 2.5. If $[x, y]^{q}=1$ for any $x, y \in G$, then $L_{p}(G)$ satisfies the identity $f \equiv 0$.

\section{Proof of the Main Result}

We start this section by citing a result of B. Hartley that will be used later on.

Theorem 3.1 (Hartley, [4]). Let $G$ be an n-generator finite solvable group of Fitting height $h$. There exists a constant $N(h, n)$ depending only on $h$ and $n$ such that each element of $G^{\prime}$ can be expressed as a product of at most $N(h, n)$ commutators.

The proof of the following lemma was suggested by A. Mann.

Lemma 3.2. Let $\pi$ be a set of primes, $G$ a finite group in which all commutators are $\pi$-elements. Then $G^{\prime}$ is a $\pi$-group.

Proof. Assume that the result is false and let $G$ be a counterexample of minimal possible order. Obviously $G$ has no non-trivial normal $\pi$-subgroups. If $r$ is a prime divisor of $|G|$ with $r \notin \pi$ and if $R$ is a Sylow $r$-subgroup of $G$, then $N_{G}(R)=C_{G}(R)$. Hence by the Burnside's Theorem [3, Theorem 7.4.3] $G$ has a normal $r$-complement $K$. By induction $K^{\prime}$ is a normal $\pi$-subgroup of $G$, whence $K^{\prime}=1$. But then $K$ and hence $G$ is a $\pi^{\prime}$-group, a contradiction.

Lemma 3.3. Let $n$ be a positive integer, $G$ a finite n-generator group such that $[x, y]^{q}=1$ for any $x, y \in G$. Then there exists an $\{n, q\}$-bounded number $\mu$ such that the exponent of $G^{\prime}$ divides $p^{\mu}$. 
Proof. By the previous lemma $G^{\prime}$ is a $p$-group, so $G$ is solvable and has Fitting height at most 2. Let $x \in G^{\prime}$ and $N(2, n)$ be as in Theorem 3.1. Theorem 3.1 says that $x$ belongs to some subgroup generated by at most $N(2, n)$ commutators. Let $m$ be the minimal number satisfying the condition that there exist commutators $a_{1}, a_{2}, \ldots, a_{m}$ such that $x \in\left\langle a_{1}, a_{2}, \ldots, a_{m}\right\rangle$. We set $H=\left\langle a_{1}, a_{2}, \ldots, a_{m}\right\rangle$. Of course $H$ is a $p$-group. Since $\left[x_{1}, x_{2}\right]^{q}=1$ for all $x_{1}, x_{2} \in H$, it follows by Lemma 2.5 that $L_{p}(H)$ satisfies the identity $f \equiv 0$.

Consider an arbitrary Lie commutator $\sigma$ in $\tilde{a}_{1}, \tilde{a}_{2}, \ldots, \tilde{a}_{m}$ and let $\rho$ be the group commutator in $a_{1}, a_{2}, \ldots, a_{m}$ having the same bracketage as $\sigma$. The definition of $L_{p}(H)$ yields that either $\sigma=0$ or $\sigma=\tilde{\rho}$. Since $\rho^{q}=1$, Lemma 2.3 implies that $\sigma$ is ad-nilpotent of index at most $q$. Theorem 2.2 now says that $L_{p}(H)$ is nilpotent of class depending only on $f, m, p$ and $q$, hence on $n$ and $q$. Combining this with Lemma 2.4 we conclude that there exists an $\{n, q\}$-bounded number $r$ such that $H$ can be written as a product of at most $r$ cyclic subgroups each of order $\leq q$. Therefore $H$ is of order at most $q^{r}$ and, in particular, the exponent of $H$ is $\{n, q\}$ bounded.

Theorem 3.4. Let $G$ be a residually finite group in which $[x, y]^{q}=1$ for all $x, y \in$ $G$. Then the derived group $G^{\prime}$ of $G$ is locally finite.

Proof. Let $S$ be any finite subset of $G^{\prime}$. Obviously we can choose a finitely generated subgroup $K$ of $G$ such that $S \subseteq K^{\prime}$. Let $K$ be $n$-generator. Then, by Lemma 3.3 , the derived group of every finite quotient of $K$ has $\{n, q\}$-bounded exponent. Since $K$ is residually finite, we conclude that $K^{\prime}$ is of finite (even $\{n, q\}$-bounded) exponent. By the positive solution to the Restricted Burnside Problem [9], [10] any residually finite group of finite exponent is locally finite. Therefore $K^{\prime}$ is locally finite and $S$ generates a finite subgroup. The proof is complete.

\section{REFERENCES}

[1] S. I. Adian, The Burnside Problem and Identities in Groups, Nauka, Moscow, 1975.

[2] Yu. A. Bakhturin, Identities in Lie algebras, Nauka, Moscow, 1985. MR 86k:17015

[3] D. Gorenstein, Finite Groups, Harper and Row, New York, 1968. MR 38:229

[4] B. Hartley, Subgroups of finite index in profinite groups, Math. Z. 168 (1979), 71-76. MR 80k:20028

[5] B. Huppert, N. Blackburn, Finite Groups II, Springer Verlag, Berlin, 1982. MR 84i:20001a

[6] S. V. Ivanov, The free Burnside groups of sufficiently large exponents, Int. J. Algebra Comput. 4(1994), 1-308. MR 95h:20051

[7] M. Lazard, Sur les groupes nilpotents et les anneaux de Lie, Ann. Sci. École Norm. Supr. 71 (1954), 101-190. MR 19:529b

[8] J.S. Wilson and E. Zelmanov, Identities for Lie algebras of pro-p groups, J. Pure Appl. Algebra, 81 (1992), 103-109. MR 93m:17004

[9] E. Zelmanov, The solution of the restricted Burnside problem for groups of odd exponent, Math. USSR Izv. 36 (1991), 41-60. MR 91i:20037

[10] E. Zelmanov, The solution of the restricted Burnside problem for 2-groups, Math. Sb. 182 (1991), 568-592. MR 93a:20063

[11] E. Zelmanov, Nil Rings and Periodic Groups, The Korean Math. Soc. Lecture Notes in Math., Seoul, 1992. MR 94c: 16027

Department of Mathematics, University of Brasilia, 70910-900 Brasilia - DF, Brazil

E-mail address: pavel@ipe.mat.unb.br 